\title{
Numerical analysis on thermal conductivity of poly-mineral rock*
}

\author{
Huimin Jing Xiangwei Yu Huai Zhang and Yaolin Shi ${ }^{*}$ \\ Laboratory of Computational Geodynamics, Graduate University of Chinese Academy of Sciences, Beijing 100049, China
}

\begin{abstract}
In order to explore the thermal conductivity of the natural poly-mineral rock, numerical tests of rock models with randomly-distributed components were conducted and compared with each other. Elaborately designed Monte Carlo method was adopted to ingratiate the requirement of the random characteristics of grain size and the grains' spatial distribution. This requirement was fulfilled by clustering the randomly generated unstructured tetrahedral elements in full three dimensions. Natural rocks are consisted of randomly distributed crystal particles or intergranular minerals. Our primary results verify that the thermal conductivity of the rock is strongly sensitive to the ingredients' volume fraction and their spatial distribution. Furthermore, we proved that, in order to reduce the measurement error to a reasonable range, the numerical specimen must be large enough or include sufficient number of mineral particles. Our numerical test results are in accordance with a variety of empirical formulas which are currently employed in petrology.
\end{abstract}

Key words: thermal conductivity; poly-mineral; numerical test; finite element method CLC number: P574.1 Document code: A

\section{Introduction}

Thermal conductivity is one of the major thermodynamic coefficients of the rock when the numerical investigation of the long-term Coulomb stress migration is carried out. Coulomb stress is now widely accepted as the crucial criteria to evaluate the characteristics of seismic activities in specific regions (Stein et al, 1997; Lin and Stein, 2004). It also has a promising future in its application to short-term earthquake forecast with essential input of real on-site geological constrains in Chinese mainland, such as western Sichuan and Yunnan area (Zhang et al, 2009). During the evolution of the thermal structure of lithosphere or thermal evolution history in deep mantle, the interior heat of the earth is transmitted to the earth's surface by two major mechanisms: advection and conduction. And conduction is the predominant mode of heat transfer in lithosphere, accompanied by the slow deformation of the plates (Clauser, 2009). Various geophysical parameters of lithosphere including the state of rock rheology, thermal structure and heat flow are all closely related to the

\footnotetext{
* Received 29 October 2009; accepted in revised form 22 January 2010; published 10 June 2010.

* Corresponding author. e-mail: shiyl@gucas.ac.cn

(C) The Seismological Society of China and Springer-Verlag Berlin Heidelberg 2010
}

thermal conductivity characteristics of the lithosphere rocks (Abdulagatov et al, 2006). In recent years, the study on the rock's thermal conductivity is one of the important issues to petrophysicist (Liu et al, 2005; Aurangzeb et al, 2006, 2007; Aurangzeb and Maqsood, 2007; Davis et al, 2007; Jessop, 2007).

Many types of advanced equipments for the measurement of rock's thermal conductivity are well developed (Ou et al, 2003; EKO, 2007). With the advancement of the instruments and the improvement of technology, systematical measurement error from equipment is becoming decreased gradually. However, it is still time-consuming and also very expensive to perform these kinds of measurements in the laboratory (Zhao et al, 1995; Liu et al, 2005). And those instruments employed also have their own technical limitations to simulate the actual high-temperature and high-pressure environment of the realistic rocks in the deep lithosphere and mantle. The major obstacles may come from those complex factors that can affect the rock's thermal conductivity. Some of them cannot be easily isolated from each other because of the nonlinear characteristics of these factors or physical parameters. Furthermore, the measurement processes are sensitive and susceptible to environmental changes, which makes it very difficult to maintain the stability of the experiment results. 
The natural occurrence of rocks is almost anisotropic, heterogenic, and multiphase (Peng and Cheng, 1999). Therefore, the granular scale effect and the component fraction effect inevitably exist during the measurement of thermal conductivity of the rock. This is basically why the various instruments must have their own specifications on the grain size and component fraction of the rock specimens. The rock specimens must be typical and representative for the natural rocks. The rock's thermal conductivity varies with the proportion and grain structure of its components (Chen and Huang, 2001). Thus we need a couple of rock samples to get the statistic effective thermal conductivity of the rocks, which is not easy to be put into practice.

In view of the difficulties mentioned above, it is not an easy task to obtain large quantities and statistical regularity of thermal conductivity values barely from laboratories. Under such circumstances, many investigators from the petrologic and geodynamical communities are now seeking to use numerical tests to replace the traditional laboratory measurement tests (Haghighi et al, 1990; Ramani and Vaidyanathan, 1995; Cheng et al, 2005; Jessop, 2007; Zhang et al, 2007). The numerical simulation of the two-phase and multiphase media is becoming one hot issue as well. Lv et al (2006) and Huai et al (2007) analyzed the effective thermal conductivity of fractal porous media by using finite volume method. Usowicz et al (2006a, b) and Zhang et al (2007) predicted the terrestrial soil with water content or air-filled porosity thermal conductivity. Wang et al (2007) investigated the spatial-scale effect on the effective thermal conductivity of porous media, and Grandjean et al (2006) calculated the thermal conductivity of porous ceramics based on micrographs by numerical methods. There are a variety of theoretical and empirical models as well (Progelhof et al, 2004; Carson, 2006). Most of those models are based on simplified cases. In those models, the shape of the specimen is either cubic or sphere. But the actual rocks are composed of irregular shapes of components. In our simulation, we use unstructured tetrahedral mesh in order to imitate the realistic plain rocks.

This paper firstly put forward our numerical test method to simulate the thermal conduction process of the given rock specimens, and then utilized the Monte Carlo statistical method to analyze the calculated data to determine the specific values of overall thermal conductivity of the rock specimen. Finite element method is used in our numerical tests. Comparison between the numerical results and empirical formula was also given to verify the correction of our models and the reliability of empirical formula as well.

\section{Principles and numerical model}

\subsection{Principles and equations}

Thermal conductivity $K$ is the physical property of a material, which characterizes its capacity to conduct the quantity of the heat across the unit area during the unit time interval. Ordinary rock is generally composed of a variety of minerals or their mixtures, and its thermal conductivity is an important physical property. There are a couple of ways to measure the rock's thermal conductivity. Each of them is only suitable for limited types of the rocks, depending on their thermal properties and existing temperature range. Apparatus for measuring thermal conductivity of rocks are generally divided into two catalogs: steady-state and non-steady state. The former requires a relatively large specimen size and the heat flow must be perpendicular to the horizontal area of the specimen. The entire system needs a stable thermal equilibrium, which can guarantee a small measurement error; whereas, the latter is suitable for the small size specimen. But its measurement accuracy is lower compared with that of the steady-state method (Ou et al, 2003).

Supposing that the thermal conductivity of each mono-mineral rock is known, we take the overall thermal conductivity of one poly-mineral rock as our test value. The finite element and Monte Carlo statistics methods are used to calculate and analyze the thermal conductivity of the poly-mineral rock. The steady-state heat conduction equation of poly-mineral rock can be expressed as

$$
-\nabla \cdot K(c, r, s) \nabla T=p
$$

where $K$ denotes the thermal conductivity, $T$ is the temperature, $c$ stands for the compositions of the rock, $r$ for the volume fraction of the components, and $p$ for the heat source term, respectively. The $s$ can be defined as the scale effect that will be fully discussed in section 3 .

The relationship between the temperature gradient and heat flux $q$ obeys the Fourier's law.

$$
q=-k \nabla T
$$

In this study, we employ the finite element method to solve equation (1). We use finite element program generator (FEPG) with the personal pre and postproces- 
sor GiD to build numerical model to simulate experiment of thermal conductivity measurement at laboratory.

The spatial structure of minerals affects the rock thermal conductivity greatly (Grandjean et al, 2006), which can be best described by two kinds of end-member cases: serial mode (the left in Figure 1) and parallel mode (the right in Figure 1). Given the same volume fraction of the two components of the dual-material rock, different types of spatial structures lead to different thermal conductivity values, which can be formulated by serial equation (3) and parallel equation (4), respectively (Progelhof et al, 2004; Carson, 2006; Carson et al, 2006):

$$
\begin{aligned}
& K=\frac{K_{1}+K_{2}}{2}, \\
& K=\frac{2 K_{1} K_{2}}{K_{1}+K_{2}},
\end{aligned}
$$

where $K$ stands for the effective thermal conductivity of the rock, $K_{1}$ and $K_{2}$ for the thermal conductivity of the first and the second component.
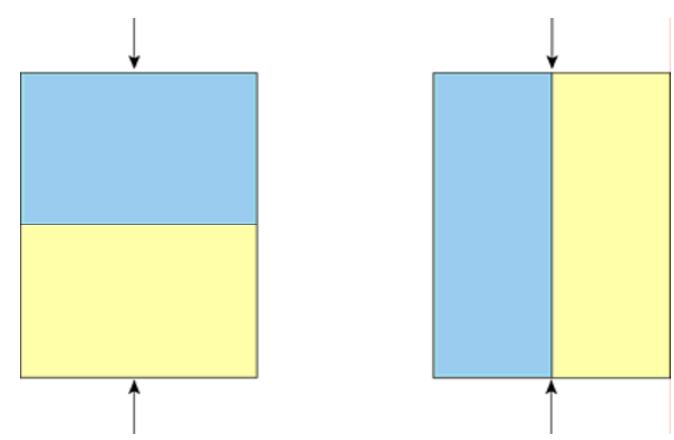

Figure 1 Two end-member modes of the dual-material rock's thermal conductivity. The left shows that the spatial structure of two kinds of mineral components in the rock is distributed in series, and the right shows that it is distributed in parallel.

In poly-mineral rocks, the proportion of different components and the spatial structure of mineral grains or particles are predominant factors that affect their thermal conductivity. Rocks under natural conditions are mostly consisted of a variety of minerals mixed together randomly. Hence a cluster of numerical specimens with randomly distributed mineral grains are needed.

\subsection{Numerical model and generation of randomly distributed meshes}

We use finite element program generator (FEPG) to generate the source program. Numerical specimen is in the shape of a cylinder. Temperatures of $333 \mathrm{~K}$ and $283 \mathrm{~K}$ are imposed as boundary conditions on the upper and lower surface. The cylindrical surface is adiabatic.
The steady-state temperature field is obtained from equation (1) by the finite element method. The overall thermal conductivity $K$ of the rock can be calculated by substituting the value of heat flux $q$ to equation (2), afterwards.

The prototype of this model is one mixture of two kinds of water-bearing limestones with different volume fraction. The thermal conductivity of these two kinds of media is assumed to be $K_{\mathrm{s}}=3.0 \mathrm{~W} /(\mathrm{K} \cdot \mathrm{m})$ and $K_{\mathrm{W}}=1.0$ $\mathrm{W} /(\mathrm{K} \cdot \mathrm{m})$. The subscripts ' $\mathrm{s}$ ' and ' $\mathrm{w}$ ' mean the medium contents more solid or water respectively. The volume fraction of two components is pre-assigned while the spatial distribution of their particles is randomly distributed. In our numerical experiments with randomly distributed unstructured tetrahedral grid model, assuming that a number of tetrahedral meshes form a mineral gain (particle clusters), thermal conductivity value can be calculated by equations (1) and (2). Basically, through sufficiently refinement of tetrahedral meshes, our numerical calculation results can converge to the actual rock, as shown in Figure 2.

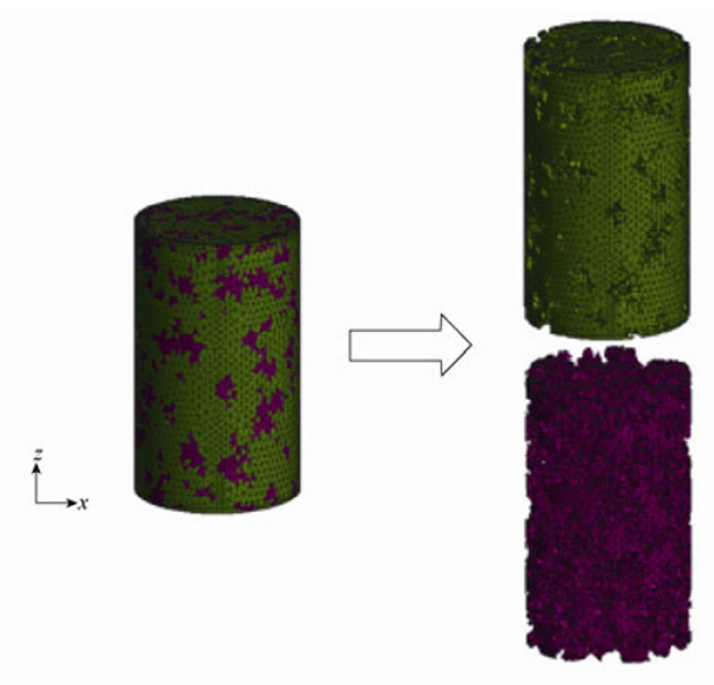

Figure 2 The randomly distributed spatial granularity specimen consisted of two kinds of mineral components. The figure shows our numerical simulation model created by randomly mixed two kinds of minerals. The left shows the specimen consisted of two kinds of components and their spatial distribution. Each color represents one mineral. The right shows their respective randomly distributed spatial configurations of these two kinds of mineral compositions.

Generally speaking, if the numerical specimen is large enough to contain abundant and randomly distributed mineral gains, it is possible to gain an approximate average result to the realistic rock. However, this is un- 
reasonable because the numerical specimen actually does not contain enough particles as natural rocks due to the huge amount computing power required. In our numerical experiments, this difficulty was overcome by Monte Carlo statistical method over a cluster of numerical tests.

\section{Calculation and result analysis}

\subsection{Calculation}

During our numerical experiments of one dualmaterial rock, the volume fraction of one component varies from 0.05 to 0.95 gradually, and the volume fraction of another component changes from 0.95 to 0.05 accordingly. A cluster of numerical tests under different volume fractions and randomly distributed spatial configurations were carried out to calculate their respective $K$ values. Then Monte Carlo method was utilized to analyze the results to obtain the statistical value of $K$. Table 1 displays our experimental conditions and results.

\subsection{Result analysis}

\subsubsection{Effect of the volume fraction}

The mineral grains are consisted of a cluster of tetrahedral cells (meshes). The total number of rock's grains within one numerical experiment specimen, to a large extend, affects the spatial distribution as well as the value of $K$ as the final result. The total number of the tetrahedral meshes is equal to or greater than that of the mineral gains, which means that one or more tetrahedral meshes can form one mineral grain. Therefore, in our numerical tests, we used $2.0 \times 10^{4}$ and $1.5 \times 10^{5}$ tetrahedral meshes to form $1.0 \times 10^{3}, 7.0 \times 10^{3}, 2.0 \times 10^{4}, 4.0 \times 10^{4}$ and $6.0 \times 10^{4}$ mineral grains, respectively. Figure 3 shows the relationship between the distribution of $K$ and the number of tetrahedral meshes.

If the volume fraction of one component is given, randomly distributed material gains can be generated. From equations (3) and (4) we can see that, the spatial distribution of one material component affects the value of $K_{\text {eff }}$ greatly. Therefore, a cluster of numerical tests with components in different spatial distribution but the same volume fraction must be carried out. Finally, Monte Carlo method was employed to generate the statistical value of $K_{\text {eff. }}$

Under the case with the same volume fraction, we carried out 300 numerical tests with randomly distributed spatial configurations of these two kinds of mineral compositions. All the $K_{\text {eff }}$ values obtained were plotted in Figure 3. Figure 3a shows the distribution of $K_{\text {eff }}$
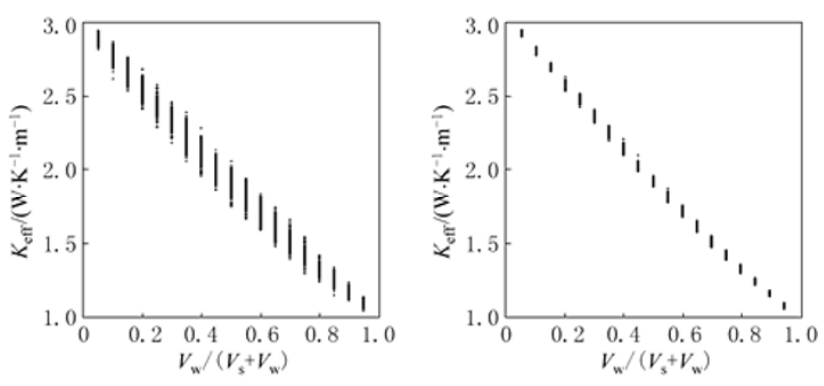

Figure 3 Distribution of $K_{\text {eff }}$ with volume fraction $V_{\mathrm{w}} /\left(V_{\mathrm{s}}+V_{\mathrm{w}}\right)$ increasing from 0.05 to 0.95 . (a) $1.0 \times 10^{3}$ grains are contained in the specimen; (b) $7.0 \times 10^{3}$ grains are in the specimen.

when $1.0 \times 10^{3}$ grains are contained in the specimens. Figure $3 \mathrm{~b}$ shows the distribution of $K_{\text {eff }}$ with $7.0 \times 10^{3}$ grains in the specimens.

From Figure 3, we can conclude that the volume fraction of the component has a significant influence on the value of $K_{\text {eff }}$ With the volume fraction of the second kind of component increasing, the $K_{\text {eff }}$ value of the entire rock specimen varies from the thermal conductivity of the first component $\left(K_{\mathrm{s}}\right)$ gradually to that of the second component $\left(K_{\mathrm{w}}\right)$. Both Figures 3 and 4 indicate that the volume fraction of the particle should up to a critical value in order to significantly improve the effective thermal conductivity, which agrees well with the percolation theory (Zhang et al, 2005).

Furthermore, the total number of mineral grains contained in the numerical specimen has great impact on the result of finite element calculation. When the specimen contains less number of mineral grains, the calculated $K_{\text {eff }}$ values fluctuate considerably and their distribution is more decentralized. On the contrary, when the specimen contains enough number of mineral grains, there are less fluctuations and the obtained test results are relatively concentrated, as shown in Figure 4.

In Figure 4, each subgraph corresponds to one volume fraction case. Since the randomly distributed grain models have been used, the experimental values obtained from these numerical tests must obey the Gaussian distribution. At the same time, the peak value of Gaussian distribution corresponds to the average value of $K_{\text {eff }}$ under certain volume fraction. Figure 4 clearly shows that the results of our numerical tests and their distribution fit perfectly with Gaussian distribution curve. It can be seen apparently that, given the same volume fraction, the more numerical grains are utilized in the specimens, more concentrated the peak values of $K_{\text {eff }}$ are. 
Table 1 The experimental conditions and calculation results

\begin{tabular}{|c|c|c|c|c|c|c|}
\hline $\begin{array}{c}\text { Case } \\
\text { number }\end{array}$ & $K_{\mathrm{s}} / K_{\mathrm{w}}$ & $\begin{array}{l}\text { Element number } \\
\qquad / 10^{4}\end{array}$ & $\begin{array}{l}\text { Mineral grain } \\
\text { number } / 10^{3}\end{array}$ & $\begin{array}{l}\text { Volume fraction } \\
\qquad V_{\mathrm{w}} /\left(V_{\mathrm{s}}+V_{\mathrm{w}}\right)\end{array}$ & $\begin{array}{c}K_{\text {eff }} \\
/\left(\mathrm{W} \cdot \mathrm{K}^{-1} \cdot \mathrm{m}^{-1}\right)\end{array}$ & $\begin{array}{l}\text { Standard deviation } \\
\qquad /\left(\mathrm{W} \cdot \mathrm{K}^{-1} \cdot \mathrm{m}^{-1}\right)\end{array}$ \\
\hline$a-1$ & $3: 1$ & 2 & 1 & 0.05 & 2.88241 & 0.02452 \\
\hline$a-2$ & $3: 1$ & 2 & 1 & 0.10 & 2.76532 & 0.03570 \\
\hline$a-3$ & $3: 1$ & 2 & 1 & 0.15 & 2.64997 & 0.04106 \\
\hline$a-4$ & $3: 1$ & 2 & 1 & 0.20 & 2.53500 & 0.04679 \\
\hline$a-5$ & $3: 1$ & 2 & 1 & 0.25 & 2.42870 & 0.04969 \\
\hline$a-6$ & $3: 1$ & 2 & 1 & 0.30 & 2.31771 & 0.04996 \\
\hline$a-7$ & $3: 1$ & 2 & 1 & 0.35 & 2.21256 & 0.05697 \\
\hline$a-8$ & $3: 1$ & 2 & 1 & 0.40 & 2.10052 & 0.04859 \\
\hline$a-9$ & $3: 1$ & 2 & 1 & 0.45 & 1.99864 & 0.05018 \\
\hline$a-10$ & $3: 1$ & 2 & 1 & 0.50 & 1.89338 & 0.05562 \\
\hline$a-11$ & $3: 1$ & 2 & 1 & 0.55 & 1.79679 & 0.05131 \\
\hline$a-12$ & $3: 1$ & 2 & 1 & 0.60 & 1.69202 & 0.05109 \\
\hline$a-13$ & $3: 1$ & 2 & 1 & 0.65 & 1.60064 & 0.04551 \\
\hline$a-14$ & $3: 1$ & 2 & 1 & 0.70 & 1.50839 & 0.04285 \\
\hline$a-15$ & $3: 1$ & 2 & 1 & 0.75 & 1.41272 & 0.04151 \\
\hline$a-16$ & $3: 1$ & 2 & 1 & 0.80 & 1.32354 & 0.03326 \\
\hline$a-17$ & $3: 1$ & 2 & 1 & 0.85 & 1.23913 & 0.03057 \\
\hline$a-18$ & $3: 1$ & 2 & 1 & 0.90 & 1.15717 & 0.02310 \\
\hline$a-19$ & $3: 1$ & 2 & 1 & 0.95 & 1.07567 & 0.01729 \\
\hline$b-1$ & $3: 1$ & 15 & 7 & 0.05 & 2.88698 & 0.00867 \\
\hline$b-2$ & $3: 1$ & 15 & 7 & 0.10 & 2.77344 & 0.01160 \\
\hline$b-3$ & $3: 1$ & 15 & 7 & 0.15 & 2.65957 & 0.01310 \\
\hline$b-4$ & $3: 1$ & 15 & 7 & 0.20 & 2.54810 & 0.01398 \\
\hline$b-5$ & $3: 1$ & 15 & 7 & 0.25 & 2.43784 & 0.01648 \\
\hline$b-6$ & $3: 1$ & 15 & 7 & 0.30 & 2.33068 & 0.01607 \\
\hline$b-7$ & $3: 1$ & 15 & 7 & 0.35 & 2.22204 & 0.01765 \\
\hline b-8 & $3: 1$ & 15 & 7 & 0.40 & 2.11677 & 0.01739 \\
\hline b-9 & $3: 1$ & 15 & 7 & 0.45 & 2.01207 & 0.01675 \\
\hline$b-10$ & $3: 1$ & 15 & 7 & 0.50 & 1.90786 & 0.01507 \\
\hline$b-11$ & $3: 1$ & 15 & 7 & 0.55 & 1.80642 & 0.01669 \\
\hline$b-12$ & $3: 1$ & 15 & 7 & 0.60 & 1.70624 & 0.01457 \\
\hline$b-13$ & $3: 1$ & 15 & 7 & 0.65 & 1.61003 & 0.01532 \\
\hline b-14 & $3: 1$ & 15 & 7 & 0.70 & 1.51566 & 0.01503 \\
\hline b-15 & $3: 1$ & 15 & 7 & 0.75 & 1.42075 & 0.01374 \\
\hline b-16 & $3: 1$ & 15 & 7 & 0.80 & 1.33317 & 0.01148 \\
\hline b-17 & $3: 1$ & 15 & 7 & 0.85 & 1.24604 & 0.00978 \\
\hline b-18 & $3: 1$ & 15 & 7 & 0.90 & 1.16118 & 0.00871 \\
\hline b-19 & $3: 1$ & 15 & 7 & 0.95 & 1.07974 & 0.00576 \\
\hline c-1 & $3: 1$ & 15 & 1 & 0.10 & 2.76007 & 0.02798 \\
\hline $\mathrm{c}-2$ & $3: 1$ & 15 & 7 & 0.10 & 2.77344 & 0.01160 \\
\hline $\mathrm{c}-3$ & $3: 1$ & 15 & 20 & 0.10 & 2.77297 & 0.01164 \\
\hline c-4 & $3: 1$ & 15 & 40 & 0.10 & 2.77212 & 0.01068 \\
\hline$c-5$ & $3: 1$ & 15 & 60 & 0.10 & 2.77249 & 0.01052 \\
\hline$c-6$ & $3: 1$ & 15 & 1 & 0.50 & 1.87641 & 0.03514 \\
\hline $\mathrm{c}-7$ & $3: 1$ & 15 & 7 & 0.50 & 1.90786 & 0.01507 \\
\hline $\mathrm{c}-8$ & $3: 1$ & 15 & 20 & 0.50 & 1.90805 & 0.01598 \\
\hline c-9 & $3: 1$ & 15 & 40 & 0.50 & 1.90868 & 0.01556 \\
\hline $\mathrm{c}-10$ & $3: 1$ & 15 & 60 & 0.50 & 1.90852 & 0.01606 \\
\hline $\mathrm{c}-11$ & $3: 1$ & 15 & 1 & 0.90 & 1.14959 & 0.01793 \\
\hline $\mathrm{c}-12$ & $3: 1$ & 15 & 7 & 0.90 & 1.16118 & 0.00871 \\
\hline$c-13$ & $3: 1$ & 15 & 20 & 0.90 & 1.16072 & 0.00845 \\
\hline $\mathrm{c}-14$ & $3: 1$ & 15 & 40 & 0.90 & 1.15979 & 0.00798 \\
\hline c-15 & $3: 1$ & 15 & 60 & 0.90 & 1.16138 & 0.00797 \\
\hline
\end{tabular}

Note: The case number is used to distinguish different type of tests, $K_{\mathrm{s}}$ is the first component's thermal conductivity of the duel-phase mixture, while $K_{\mathrm{w}}$ is of the second component. Element number equals the number of meshes in the numerical specimens, mineral grain number is the number of mineral groups included in the specimens, volume fraction $V_{\mathrm{w}} /\left(V_{\mathrm{s}}+V_{\mathrm{w}}\right)$ is the volume ratio of the second component to the specimen, and $K_{\text {eff }}$ is the mean effective thermal conductivity of the specimens in each case. In every case, 300 tests were carried out and the standard deviations were displayed in the last column.

\subsubsection{Error analysis}

Considering the numerical and experimental value of the rock's thermal conductivity, the errors of our numerical tests lie in two aspects, i.e., the volume fraction and spatial structure of the components. Firstly, we analyzed the error of the same grains but different volume fractions. In this case, the total number of grains in the numerical specimen was fixed, but the volume fraction 
was changed so as to get the regularity of the error variation with the volume fractions. Secondly, we analyzed the error under the case with the same volume fraction but with different total numbers of the grains. In this case, the volume fraction was fixed to observe the regularity of the error variation with the change of the total number of grains contained in the specimen. The results are shown in Figure 5.

Figure 5 shows the errors variation with volume fraction when the total number of grains in the specimens is taken as $1.0 \times 10^{3}$ and $7.0 \times 10^{3}$. It is obvious that, for the same volume fraction, the error of the former is larger than that of the latter. Furthermore, when the volume fraction is getting close to 0 or 1.0 , the error tends to decrease rapidly. Regarding the effect of granularity degrees, the more total number of grains used in our numerical test specimens, the smaller the error becomes.
Especially, when the volume fractions of two components are similar, this phenomenon is even more obvious.

Because the sources of the error are uncertain these phenomena still need to be fully addressed in the future. However, some reasons can now be briefly proposed from our numerical tests hereby. According to the statistical theory, when specimen contains two kinds of components with similar number of grains, then more kinds of spatial distribution models may be generated in our numerical tests. This will lead to the largest tolerance while no enough number of numerical tests was performed. When the total number of grains contained in the specimen is increased, such deficits mentioned above will be reduced gradually. Thereby, the error of numerical tests diminishes correspondingly.
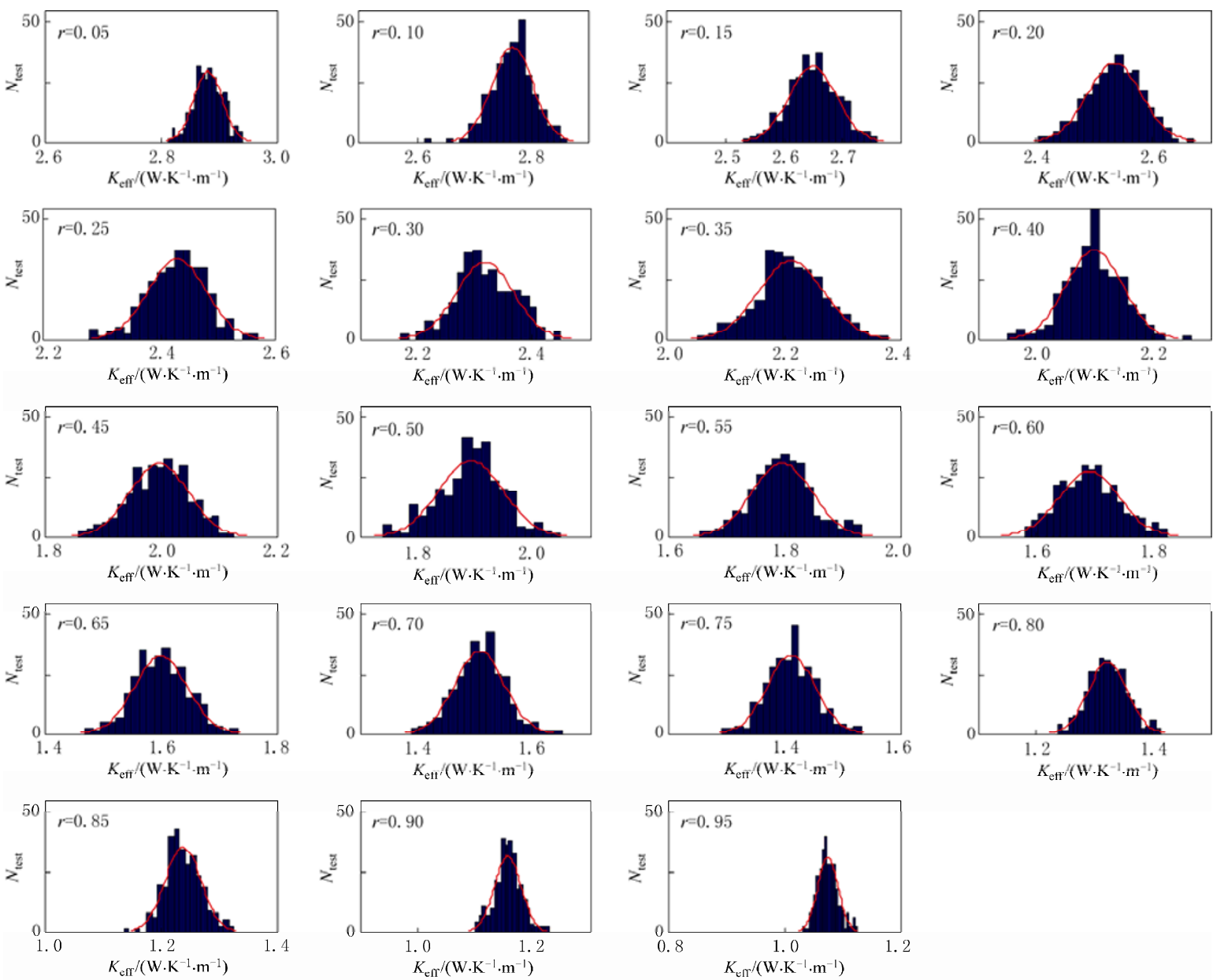

(a)

Figure 4 Gaussian distribution of $K_{\text {eff }}$ with volume fraction of the second component, $r=V_{\mathrm{w}} /\left(V_{\mathrm{s}}+V_{\mathrm{w}}\right)$, increasing from 0.05 to 0.95 gradually, where $N_{\text {test }}$ is the number of the tests. (a) $1.0 \times 10^{3}$ granularity degree $\left(1.0 \times 10^{3}\right.$ total grains of two material components) is contained. 

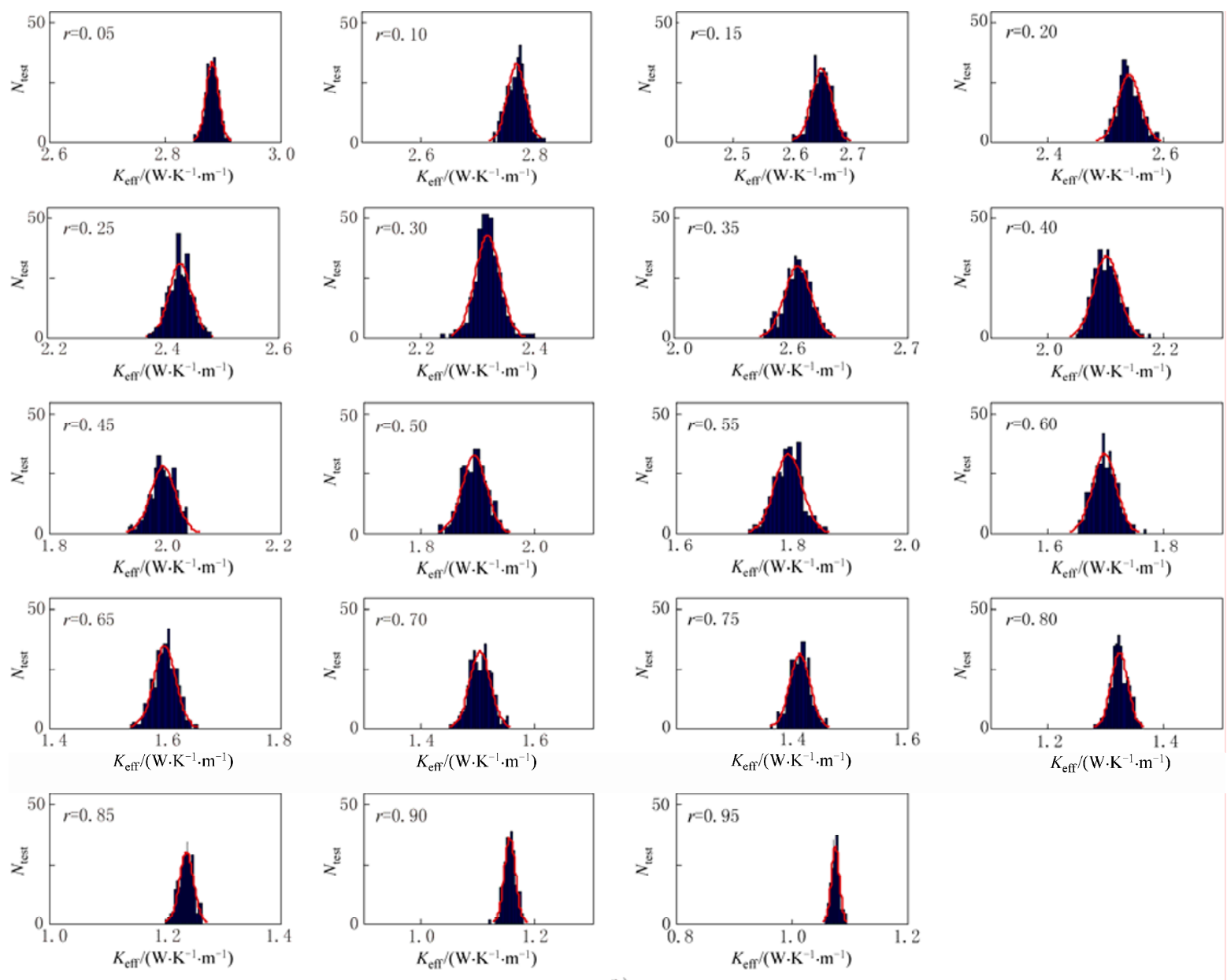

(b)

Figure 4 Gaussian distribution of $K_{\mathrm{eff}}$ with volume fraction of the second component, $r=V_{\mathrm{w}} /\left(V_{\mathrm{s}}+V_{\mathrm{w}}\right)$, increasing from 0.05 to 0.95 gradually, where $N_{\text {test }}$ is the number of the tests. (b) $7.0 \times 10^{3}$ granularity degree $\left(7.0 \times 10^{3}\right.$ total grains of two material components) is contained.

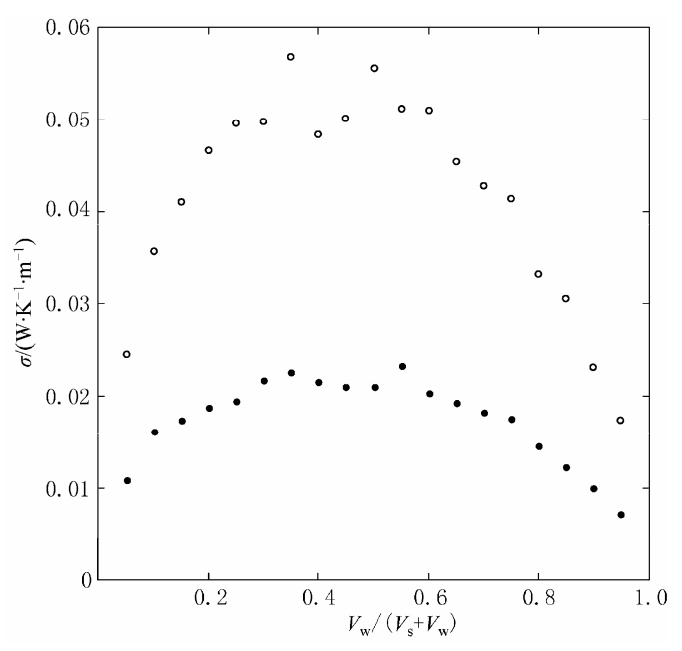

Figure 5 Distribution of standard deviation of thermal conductivities when the sample contains $1.0 \times 10^{3}$ grains (denoted by circles) and $7.0 \times 10^{3}$ grains (denoted by dots).

\subsubsection{Effects of granularity}

Physically, most of the natural occurrences of rocks are multi-phase mixtures with anisotropy and heterogeneity (Peng and Cheng, 1999). Therefore the presence of scale effect and component proportional (volume fraction) effect in the determination of the rock's thermal conductivity are inevitable. This is actually the reason why the existing measuring instruments require different size of the rock specimen. Then one question is raised: what size of the rock specimen under a certain percentage of components can result in a reasonable value of thermal conductivity for the natural rock? This problem can also be addressed through numerical experiments.

For different rocks, the mineral compositions are of different grain sizes and volume fractions; moreover, the grain size is irregular. Under such cases, we select the volume ratio and the average size of mineral grains as the criteria to explore their effects. The volume ratio is 
the ratio between specimen's volume and the average grain volume. Our numerical experiments were carried out under a fixed ratio between two kinds of mineral compositions. Thus we can analyze the effects of the volume ratio of the specimen and the average size of mineral grains on the experimental errors. The scale effect in the determining thermal conductivity of rocks was studied by our numerical tests in detail, and the experiment results are shown in Figure 6.
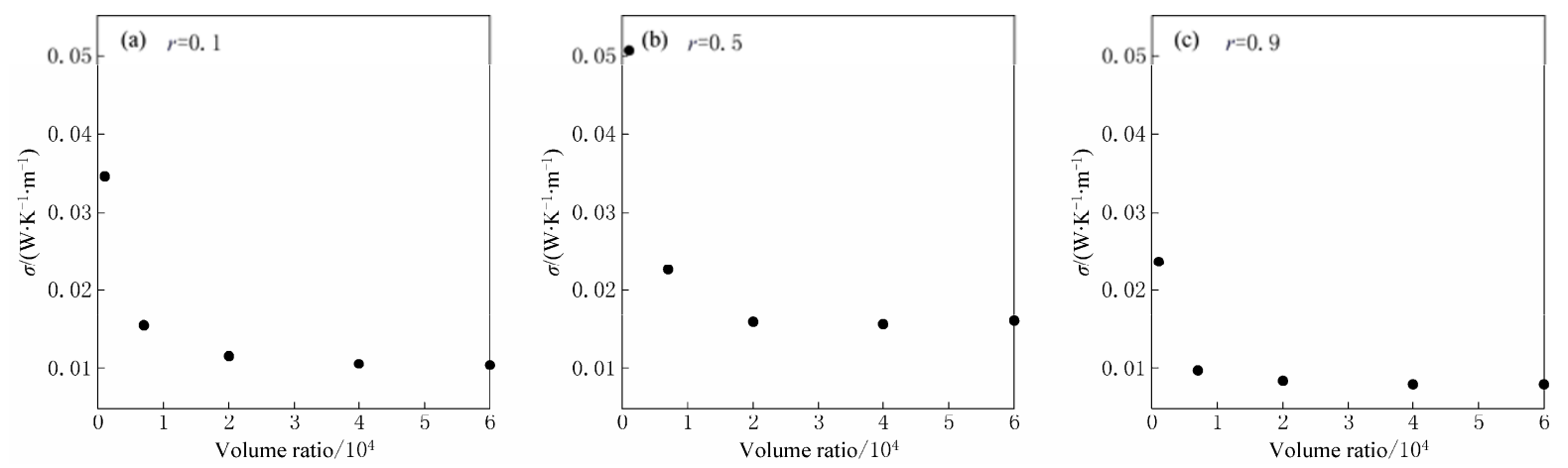

Figure 6 Error of thermal conductivity $(\sigma)$ versus different volume ratios when the volume fraction, $r=V_{\mathrm{w}} /\left(V_{\mathrm{s}}+V_{\mathrm{w}}\right)$, is taken as 0.1 (a), 0.5 (b) and 0.9 (c). The volume ratio is defined as the ratio between specimen's volume and the average grain volume.

In the three cases shown in Figure 6, the ratio of the specimen's volume to the average volume of mineral grains denoted by volume ratio are equal to $1.0 \times 10^{3}$, $7.0 \times 10^{3}, 2.0 \times 10^{4}, 4.0 \times 10^{4}$ and $6.0 \times 10^{4}$ respectively. The error distribution of these three figures shows the same tendency, i.e., the error decreases with the increasing of the ratio between the volume of the specimen and the average volume of the grains. When this ratio increases to a certain value, the change of the error becomes slow down. From these figures, we can see that the critical value of the ratio between the volume of the specimen and the average volume of the grains is around $1.0 \times 10^{4}$. In other words, for certain average size of mineral grains of the rock specimen, to obtain relatively small experimental error, the rock specimen should be greater than $1.0 \times 10^{4}$ times of the average size of mineral grains. Of course, the error can be further reduced through gradually increasing the volume of the specimen. But increasing the specimen volume may lead to more difficulty and costs in actual experiment. Therefore, the suggested ratio of the specimen volume to the average volume of mineral grains is around $1.0 \times 10^{4}$, which is reasonable to meet scientific accuracy and economic requirements of scientific experiments.

\section{Comparison with empirical formulas}

Thermal conductivity of the dual-phase or poly-phase mixture is related to the fraction of each component. At present, many researchers have carried out relevant research through laboratory experiment and numerical test (Lv et al, 2006; Grandjean et al, 2006; Usowicz et al, 2006a, b; Huai et al, 2007; Wang et al, 2007; Zhang et al, 2007), many empirical formulas are summarized. We drew a comparison between some selected empirical formulas and fitting curves obtained from our experimental values. The symbolic significance of the following formula is as following:

$K_{\text {eff: }}$ the effective thermal conductivity of the rock;

$K_{\mathrm{s}}$ : the first component's thermal conductivity of the dual-phase mixture;

$K_{\mathrm{w}}$ : the second component's thermal conductivity of the dual-phase mixture;

$r$ : the volume fraction of the second component accounted for the rock minerals.

Progelhof et al (2004) and Carson (2006) have summarized the calculating formulas of the thermal conductivity of multi-phase mixture. The follows are some of the formulas.

For the geometric mean model of two-phase mixture,

$$
K_{\mathrm{eff}}=K_{\mathrm{s}}^{(1-r)} K_{\mathrm{w}}^{r}
$$

For the serial model of two-phase mixture,

$$
K_{\text {eff }}=\frac{K_{\mathrm{s}} K_{\mathrm{w}}}{r K_{\mathrm{s}}+(1-r) K_{\mathrm{w}}} .
$$

For the parallel model of two-phase mixture,

$$
K_{\text {eff }}=(1-r) K_{\mathrm{s}}+r K_{\mathrm{w}} \text {. }
$$


For the Maxwell-Eucken model,

$$
K_{\text {eff }}=K_{\mathrm{s}} \frac{2 K_{\mathrm{s}}+K_{\mathrm{w}}-2\left(K_{\mathrm{s}}-K_{\mathrm{w}}\right) r}{2 K_{\mathrm{s}}+K_{\mathrm{w}}+\left(K_{\mathrm{s}}-K_{\mathrm{w}}\right) r} .
$$

And for the EMT model,

$$
\begin{gathered}
K_{\text {eff }}=\frac{1}{4}\left\{(3 r-1) K_{\mathrm{w}}+[3(1-r)-1] K_{\mathrm{s}}+\right. \\
\left.\sqrt{\left\{(3 r-1) K_{\mathrm{w}}+[3(1-r)-1] K_{\mathrm{s}}\right\}^{2}+8 K_{\mathrm{s}} K_{\mathrm{w}}}\right\} .
\end{gathered}
$$

In addition, Zhang et al (2007) studied the thermal conductivity of soil containing porous water and air. In our tests, the model is consisted of two kinds of mineral compositions; we assumed the volume fraction of the air is zero, so only the soil and porous water are considered.

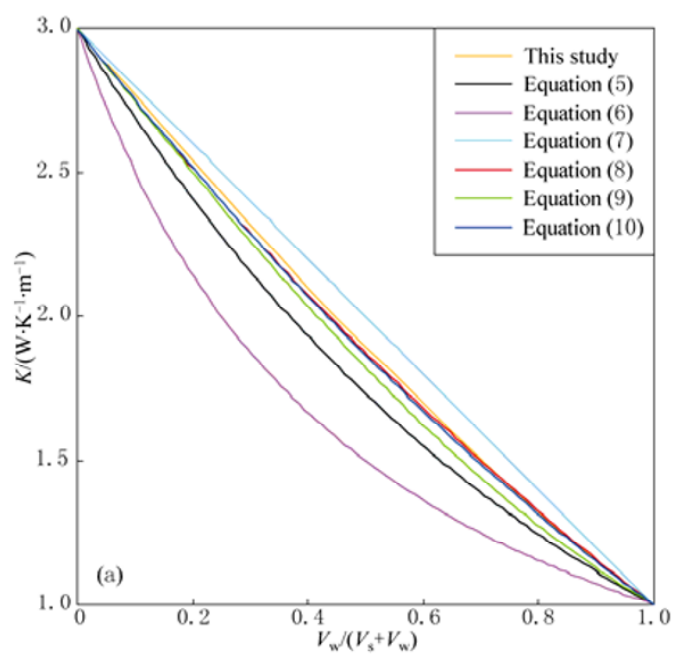

The simplified formula of the model is

$$
K_{\text {eff }}=\left[(1-r) K_{\mathrm{s}}^{0.5}+r K_{\mathrm{w}}^{0.5}\right]^{2} .
$$

Figure $7 \mathrm{a}$ shows the results from a variety of empirical formulas including our calculated results. The cyan line from equation (7) gives the upper bound of the thermal conductivity, while the lilac line which interprets equation (6) gives the lower bound. Our numerical result is between them and very close to the red line interpreting equation (8) (also seen in Figure 7b). Because equation (8) comes from a model similar to ours, in which two kinds of minerals are randomly mixed, the error between our numerical result and that from equation (8) is kindly under $2 \%$.

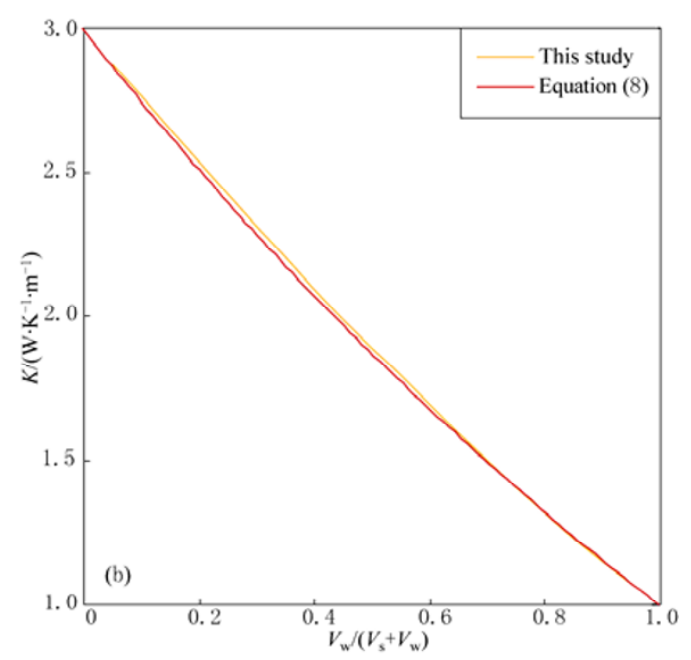

Figure 7 Comparison of our calculated results with that from a variety of empirical formulas based on some empirical formula (a) and the Maxwell-Eucken model (b).

The precision of the general thermal conductivity meters is from $1 \%$ to $5 \%$. From Table 1 , when the element number is $2.0 \times 10^{4}$, the maximum error is about $2.7 \%$; when the element number is $1.5 \times 10^{5}$, the maximum error is about $0.8 \%$. Since the largest specimen of the thermal conductivity meter commonly used is limited, the measurement error will be larger when the specimen contains less mineral grain. This means that there is another error that the thermal conductivity cannot reflect. So the larger grain of the rock requires the larger specimens. In laboratory experiment, if there are no such instruments, a huge amount of experiments are needed to get the statistical generalized value of the thermal conductivity. Furthermore, we cannot ensure that the component fraction of all the specimens used in the experiments is consistent. This will make it very dif- ficult to distinguish the source of error. In such cases, the numerical test method proposed in this paper is more feasible and efficient, and more importantly, can basically avoid these problems as well.

\section{Conclusions}

This paper proposed a numerical test method to calculate the thermal conductively, and proved its feasibility and efficiency. The numerical experiment method is based on the finite element calculation and Monte Carlo statistics method. Our analyzed results show that the thermal conductivity of the dual-mineral rock varies with the component fraction and the spatial structure. The values from our numerical tests were benchmarked with the empirical formula. Although there are a number 
of empirical formulas, each of them is suitable for a particular case; our procedure can perform efficiently under the more complex situations, for example, the poly-phase mixtures with complex distribution of different components. In this case, all the existing empirical formulas are invalid. In addition, we proved numerically that the sufficient number of grains in the experiments must be ensured and the critical ratio value of the specimen volume to the average volume of the grains is around $1.0 \times 10^{4}$. The comparison with the empirical formulas also shows the reliability of our numerical model. Especially important, our method can be further extended to the more complex cases and the other thermodynamic properties of rocks, such as their mechanical and electromagnetic properties. Our error analysis of the grain size and component fraction of the rock's mineral phases provided us with valuable guidance for the future research.

Acknowledgments This work was supported by the National Science and Technology Project (No. SinoProbe-07), National Basic Research Program of China (No. 2008cb425701), and National Natural Science Foundation of China (No. 40774049). We thank Institute of Earthquake Science, China Earthquake Administration, who provided us with the support from the collaborative project named as "Pre-study of several issues of testing ground for numerical forecast earthquakes in North China".

\section{References}

Abdulagatov I M, Emirov S N, Abdulagatova Z Z and Askerov S Y (2006). Effect of pressure and temperature on the thermal conductivity of rocks. Journal of Chemical and Engineering Data 51(1): 22-33.

Aurangzeb, Ali Z, Gurmani S F and Maqsood A (2006). Simultaneous measurement of thermal conductivity, thermal diffusivity and prediction of effective thermal conductivity of porous consolidated igneous rocks at room temperature. Journal of Physics D: Applied Physics 39(17): 3 876-3 881.

Aurangzeb, Khan L A and Maqsood A (2007). Prediction of effective thermal conductivity of porous consolidated media as a function of temperature: a test example of limestones. Journal of Physics D: Applied Physics 40(16): 4 953-4 958.

Aurangzeb and Maqsood A (2007). Modeling of the effective thermal conductivity of consolidated porous media with different saturants: A test case of gabbro rocks. International Journal of Thermophysics 28(4): 1371-1 386.

Carson J K (2006). Review of effective thermal conductivity models for foods. International Journal of Refrigeration 29(6): 958-967.

Carson J K, Lovatt S J, Tanner D J and Cleland A C (2006). Predicting the effective thermal conductivity of unfrozen, porous foods. Journal of Food Engineering 75(3): 297-307.

Chen Y and Huang T F (2001). Petrophysics. Peking University Press, Beijing, 212-216 (in Chinese).

Cheng Y B, Wu G M, Ma J H and Shen J (2005). Numerical simulation of heat transfer of horizontal column at high temperature. Ordnance Material Science and Engineering 25(2): 31-34 (in Chinese with English abstract).
Clauser C (2009). Heat transport processes in the Earth's crust. Surveys in Geophysics 30(3): 163-191.

EKO (2007). Thermal Conductivity Tester HC-110 (AUTOA). 2010-04-05, http://www.eko-usa.com/products/mt/HC/HC-110/HC-110.html.

Davis M G, Chapman D S, Van Wagoner T M and Armstrong P A (2007). Thermal conductivity anisotropy of metasedimentary and igneous rocks. $J$ Geophys Res 112: B05216, doi:10.1029/2006JB004755.

Grandjean S, Absi J and Smith D S (2006). Numerical calculations of the thermal conductivity of porous ceramics based on micrographs. Journal of the European Ceramic Society 26(13): 2 669-2 676.

Haghighi K, Irudayaraj J, Stroshine R L and Sokhansanj S (1990). Grain kernel drying simulation using the finite-element method. Trans ASAE 33(6): 1957-1965.

Huai X L, Wang W W and Li Z (2007). Analysis of the effective thermal conductivity of fractal porous media. Applied Thermal Engineering 27(17-18): 2815-2 821.

Jessop A (2008). Models of thermal conductivity of crystalline rocks. International Journal of Earth Sciences 97(2): 413-419.

Lin J and Stein R S (2004). Stress triggering in thrust and subduction earthquakes and stress interaction between the southern San Andreas and nearby thrust and strike-slip faults. J Geophys Res 109(B2): B02303, doi:10.1029/2003JB002607.

Liu D W, Gu D S, Dai T G and Henkel H (2005). Analysis and calculation of thermal conductivity of rock in deep strata. J Cent South Univ Technol 12(1): 114-119.

Lv Y G, Huai X L and Wang W W (2006). Study on the effect of micro geometric structure on heat conduction in porous media subjected to pulse laser. Chemical Engineering Science 61(17): 5717-5 725.

Ou X G, Jin Z M, Jin S Y and Xu H J (2003). Thermal conductivity of Donghai UHP eclogite and its significance for studying continental scientific drilling. Earth Science - Journal of China University of Geosciences 28: 129-136 (in Chinese with English abstract).

Peng D R and Cheng P J (1999). Studies on thermal properties and thermal conductivity of coal and rock. Coal 8: 13-19 (in Chinese).

Progelhof R C, Throne J L and Ruetsch R R (2004). Methods for predicting the thermal conductivity of composite systems: A review. Polymer Engineering and Science 16(9): 615-625.

Ramani K and Vaidyanathan A (1995). Finite element analysis of effective thermal conductivity of filled polymeric composites. Journal of Composite Materials 29: 1725-1 740.

Stein R S, Barka A A and Dieterich J H (1997). Progressive failure on the North Anatolian fault since 1939 by earthquake stress triggering. Geophysical Journal International 128(3): 594-604.

Usowicz B, Lipiec J and Ferrero A (2006). Prediction of soil thermal conductivity based on penetration resistance and water content or air-filled porosity. International Journal of Heat and Mass Transfer 49(25-26): 5010-5 017.

Usowicz B, Lipiec J, Marczewski W and Ferrero A (2006). Thermal conductivity modelling of terrestrial soil media - A comparative study. Planet Space Sci 54(11): 1 086-1 095.

Wang M, Wang J K, Pan N, Chen S Y and He J H (2007). Three-dimensional effect on the effective thermal conductivity of porous media. Journal of Physics D: Applied Physics 40(1): 260-265.

Zhang H, Wu Z, Liu J, Wang H, Yan Z and Shi Y (2009). Virtual ChuanDian A parallel numerical modeling of Sichuan-Yunnan regional strong earthquake activities: Model construction and parallel simulation. Science in China (Series D) 39(3): 260-270.

Zhang H F, Ge X S and Ye H (2005). Effectiveness of the heat conduction reinforcement of particle filled composites. Modelling and Simulation in Materials Science and Engineering 13(3): 401-412.

Zhang H F, Ge X S, Ye H and Jiao D S (2007). Heat conduction and heat storage characteristics of soils. Applied Thermal Engineering 27(2-3): 369-373.

Zhao Y X, Yang S Z, Zhang W R, Liang X R and Ma L Y (1995). An experimental study of rock thermal conductivity under different temperature and pressure. Progress in Geophysics 10(1): 104-113 (in Chinese with English abstract). 